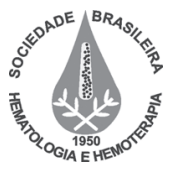

Atualização / Update

\title{
Preservação da fertilidade em pacientes com câncer
}

\section{Fertility preservation for oncologic patiens}

Daniella S. Castellotti ${ }^{1}$

Arnaldo S. Cambiaghi ${ }^{2}$

\begin{abstract}
Os avanços tecnológicos dos últimos anos na área de oncologia têm proporcionado aos pacientes tratamentos que revolucionaram a esperança de ter uma vida melhor. Jovens que tinham, muitas vezes, um futuro sombrio podem agora olhar com esperanças concretas a cura de sua doença. Entretanto, esta evolução nem sempre tem evitado o prejuízo da saúde reprodutiva causado pelas cirurgias, quimioterapias ou radioterapias, comuns a estes pacientes. Os especialistas em Hematologia e Oncologia devem ter conhecimento da existência de técnicas que podem preservar a fertilidade do paciente e estar apto a conversar sobre este tema com o mesmo. As novas técnicas de preservação da fertilidade, como o congelamento de óvulos, fragmentos de ovário, fragmentos de testículo e outras utilizadas há mais tempo - congelamento de sêmen e embriões - associados às técnicas de fertilização assistida, abrem a perspectiva para que o desejo de ter filhos após o término do tratamento não se torne uma frustração e sim uma realidade viável. Rev. Bras. Hematol. Hemoter. 2008;30(5):406-410.
\end{abstract}

Palavras-chave: Preservação da fertilidade; reprodução assistida; congelamento de óvulos; oncologia.

\section{Introdução}

Os recentes avanços na área da oncologia referentes ao diagnóstico precoce e à eficácia dos tratamentos de pacientes com câncer promovem a cura em considerável número de pessoas, muitas delas jovens, em idade reprodutiva.

Estima-se que, em 2010, um em cada 250 adultos será sobrevivente de tratamentos de câncer, pois a radioterapia, a quimioterapia, em conjunto com cirurgias, podem curar até $90 \%$ dos casos. ${ }^{1}$ Levando-se em conta não apenas a cura, mas também a qualidade de vida, a responsabilidade do médico oncologista torna-se ainda maior, pois o mesmo deve estar atento à preservação da fertilidade deste paciente ou, do contrário, um futuro muito frustrante pode acontecer.

Quanto às mulheres, calcula-se que, anualmente, 650 mil são atingidas pelo câncer invasivo e 8\% delas (52 mil) têm menos de 40 anos. Estima-se que uma a cada 52 mulheres deverá ter câncer antes dos $39 \operatorname{anos}^{2,3}$ e a cada ano mais mulheres jovens têm câncer. Também é registrado, anualmente, um aumento de $0,3 \%$ de casos de câncer no mundo. Por outro lado, o índice de cura vem aumentando $0,6 \%$ ao ano, o que gera esta preocupação em preservar a fertilidade destes pacientes. ${ }^{4}$

A radioterapia, quando for realizada no baixo abdômen, poderá danificar ou até destruir os ovários, dependendo do tamanho e da localização do tumor e da intensidade da irradiação necessária para a cura. ${ }^{5} \mathrm{O}$ mesmo pode ocorrer com a quimioterapia, ${ }^{6}$ que, dependendo das drogas utilizadas e das doses necessárias para a cura da doença, poderá, além de extinguir o tumor, prejudicar também a função ovariana. ${ }^{7}$

As cirurgias castradoras são, muitas vezes, a melhor opção para a cura da mulher, entretanto poderão definir para sempre o futuro infértil da mesma. As mesmas considerações são válidas para o homem.

${ }^{I}$ Médica do Instituto Paulista de Ginecologia, Obstetrícia e Reprodução Humana - São Paulo-SP.

${ }^{2}$ Diretor do Instituto Paulista de Ginecologia, Obstetrícia e Reprodução Humana - São Paulo-SP.

Instituto Paulista de Ginecologia, Obstetrícia e Reprodução Humana - São Paulo-SP.

Correspondência: Daniella S. Castellotti

Rua Abílio Soares, 1125 - Paraíso

04005-004 - São Paulo-SP - Brasil

Tel.: 5511 3885-4333; 5511 3884-3218

E-mail:daniella@ipgo.com.br 
Assim, é importante que o médico que trata o paciente com câncer tenha conhecimento das técnicas atuais para preservação da fertilidade, a fim de garantir, após a cura dos seus pacientes, a possibilidade de terem filhos e construírem suas famílias.

\section{Técnicas para preservação da fertilidade}

\section{No homem}

Congelamento do sêmen - É um processo realizado com técnicas bem estabelecidas e resultados confiáveis. O sêmen deverá ser coletado através da masturbação, preferencialmente em várias amostras. Será congelado a $-196^{\circ} \mathrm{C}$, armazenado por tempo indeterminado, podendo ser descongelado e utilizado no momento adequado.

Congelamento de tecido testicular - Embora o congelamento do sêmen seja uma opção simples e de fácil execução, o congelamento do tecido testicular pode oferecer uma opção a longo prazo, principalmente nos casos de alguns tumores que prejudicam a qualidade do sêmen. ${ }^{8}{ }^{89}$ É ainda uma técnica experimental, mas pode, em alguns casos, ser a única opção. Espera-se que, no futuro, com o avanço nas pesquisas para o uso de células-tronco, a técnica de congelamento testicular possa ser uma alternativa interessante.

Observação: Quando houver urgência para o início do tratamento oncológico, pelo menos uma amostra de sêmen deverá ser congelada.

Perguntas que deverão ser respondidas pelo médico ao paciente, seus pais ou responsáveis (caso seja menor de idade), antes do início do tratamento oncológico:

1) O tratamento afetará a fertilidade do homem ou do menino? Se a resposta for "sim", qual a melhor técnica para preservar a fertilidade?

2) Como e quando o paciente poderá saber se é fértil ou não após o término do tratamento? Existem testes para isto?

3) Se a fertilidade não for preservada, existem alternativas para que ele possa ter filhos futuramente?

4) Se for realizado o congelamento do sêmen ou tecido testicular, existe uma data limite para serem utilizados?

5) Haverá mudanças do desejo sexual?

6) Haverá riscos para a gestação ou para a criança quando ele tiver seus filhos?

7) Quais são as clínicas especializadas que podem indicar os melhores métodos para preservar a fertilidade?

\section{Na mulher}

As meninas nascem com um número limitado de óvulos. A quantidade de óvulos diminui gradativamente a partir da primeira menstruação até chegar na menopausa quando já não existem mais óvulos disponíveis para serem fertilizados. ${ }^{10,11}$

Novas técnicas têm trazido esperança para preservar ou recuperar a fertilidade em meninas e mulheres que são submetidas a tratamentos de câncer. Entre elas estão o congelamento de embriões, tecido ovariano, óvulos e transposição dos ovários em caso de radioterapia.

\section{Congelamento de embriões}

Através da fertilização in vitro, o ovário é estimulado com hormônios, os óvulos retirados e posteriormente fertilizados em laboratório. Formam-se os embriões, que serão congelados em nitrogênio líquido a $-196^{\circ} \mathrm{C}$, permanecendo assim por tempo indeterminado. É considerada uma boa técnica por ser eficaz e proporcionar taxas de gravidez ao redor de $30 \%-40 \%,{ }^{12}$ mas é restrita a pacientes que não necessitam de um tratamento oncológico imediato e a tumores que não são afetados por hormônios. Além disso, a mulher já deve estar com o parceiro com o qual pretende formar uma família. Outra preocupação é o fato dos embriões serem legal e eticamente considerados seres vivos e, por isto, em nenhuma hipótese, poderão ser descartados. Caso haja desinteresse por um dos membros do casal em manter os embriões congelados, ou o desejo de utilizá-los para futura gestação, eles não poderão ser exigidos pelo outro, o que pode levar, muitas vezes, a conflitos judiciais. O congelamento de óvulos e tecido ovariano não têm este compromisso.

\section{Congelamento de tecido ovariano}

Pode ser uma ótima alternativa para crianças que ainda não atingiram a puberdade e por isto não têm ainda óvulos para serem congelados, e em pacientes que não podem ser submetidos à indução da ovulação com hormônios. Através da videolaparoscopia, uma técnica cirúrgica minimamente invasiva, é retirada uma parte de um dos ovários. Este tecido é congelado, permanecendo assim até o momento adequado para ser reimplantado. Não existe um período pré-determinado. $\mathrm{O}$ tecido poderá ser fragmentado, ou não, e poderá ser reimplantado na região pélvica, sobre o outro ovário, perto das trompas (tópico), ou em locais diferentes, como parede abdominal ou braço (heterotópico). ${ }^{13}$ Nestas condições, para que ocorra gravidez, normalmente são necessários medicamentos para indução da ovulação usados habitualmente nos tratamentos de fertilização in vitro. Ainda é uma opção que oferece pequenas taxas de sucesso, mas pode ser indicada quando não houver outra alternativa mais adequada.

\section{Congelamento de óvulos}

É uma técnica muito importante por oferecer bons resultados de gravidez futura. Tem como vantagem, em relação aos embriões, o fato de serem células, e, por isto, se não forem mais desejados, poderão ser descartados. A paciente deverá ser submetida a um tratamento de indução da ovulação semelhante ao da fertilização in vitro com a retirada dos óvulos e posterior congelamento. Nestes casos, existem duas possibilidades com o mesmo fim. Se o tumor que a paciente tem necessitar de quimioterapia e puder esperar três a cinco 
semanas para o início do tratamento oncológico, receberá medicamentos para a estimulação ovariana para que haja um número maior de óvulos a serem congelados, pois um número maior garante melhores resultados no futuro. O tipo de medicação vai depender do tumor ser sensível ou não ao hormônio estrogênio, que poderá se elevar neste tipo de tratamento e piorar a evolução da doença. Entretanto, é importante saber que, para estes casos, existem estratégias adequadas para induzir a ovulação, as quais encurtam o período de indução e exposição do tumor a este hormônio. Mas, se não puder receber os hormônios convencionais, poderão ser utilizados inibidores da aromatase que podem gerar um número menor de óvulos ou até utilizar um ciclo natural sem remédios.

Em alguns casos específicos, os óvulos poderão ser maturados no laboratório por uma técnica especial (maturação in vitro) para, posteriormente, serem congelados. ${ }^{14,15}$ Desta maneira diminui-se ainda mais o tempo de exposição ao estrogênio.

\section{Transposição dos ovários}

Nas situações em que for necessária a radioterapia na região pélvica, os ovários poderão ser atingidos diretamente e ter a sua reserva ovariana prejudicada. Para se evitar esta proximidade dos ovários com as "sondas" dos aparelhos, poderá ser realizada uma cirurgia minimamente invasiva (videolaparoscopia), que colocará os ovários por trás do útero durante o período do tratamento ou em outra localização distante do local que será atingido pela radiação. Como a radioterapia não agride o útero, que servirá como "escudo" protetor dos ovários, eles estarão protegidos. ${ }^{16,17}$ Após o término do tratamento, através da mesma técnica cirúrgica os ovários poderão voltar para o local original.

Perguntas que deverão ser respondidas pelo médico a paciente, seus pais ou responsáveis (caso seja menor de idade), antes do início do tratamento oncológico:

1) O tratamento afetará a fertilidade da mulher ou menina? Se a resposta for "sim", qual a melhor técnica para preservar a fertilidade?

2) Como e quando a paciente poderá saber se é fértil ou não após o término do tratamento? Existem testes para isso?

3) Se a fertilidade não for preservada, quais são as alternativas para que ela possa ter filhos?

4) Se houver falência ovariana (menopausa pelo tratamento) quais serão os sintomas? Existe tratamento para isso?

5) Depois de terminado o tratamento, quanto tempo levará para que a menstruação retorne? Se não estiver menstruando será necessário o uso de hormônios ou contracepção se desejar evitar filhos?

6) Haverá mudanças do desejo sexual?

7) A gravidez é segura após o tratamento? Se a resposta for positiva, quanto tempo a paciente deverá esperar para ficar grávida?
8) Haverá riscos para a gestação ou para a criança caso ela fique grávida?

9) Onde o paciente poderá encontrar clínicas de reprodução humana especializadapara este tipo de tratamento?

\section{Decidindo a estratégia para a preservação da fertilidade na mulher}

\section{Avaliação inicial-Risco de Metástase - Envolvimento ovariano}

Embora a maioria dos cânceres não levem metástases para o ovário, alguns deles, como as leucemias, podem causar este tipo de envolvimento o que pode ser grave. O neuroblastoma, por exemplo, apresenta grandes chances de causar este mal, enquanto o câncer de mama tem baixo risco de metástase ovariana (Tabela 1), bem como o tumor de Wilms e o Sarcoma de Ewing. ${ }^{18}$ No câncer de colo uterino de células escamosas, o grau de envolvimento ovariano é inferior a $0,2 \% \cdot{ }^{19}$ Independentemente do risco previamente conhecido das chances de metástase ovariana, os fragmentos de ovários que serão congelados devem ter amostras para serem examinadas e confirmarem diagnóstico da ausência de células malignas.

Existem várias opções para a preservação da fertilidade em mulheres com câncer. A estratégia a ser escolhida vai depender da idade da paciente, o tempo disponível para que as medidas possam ser tomadas sem atrapalhar o o sucesso do tratamento oncológico e o tipo de câncer. O esquema a seguir ajuda a encontrar a melhor opção para a mulher:

- Radioterapia pélvica

- Maturação de óvulo in vitro

- Transplante do ovário ou fragmentos na pelve próximo ao local dos ovários

- Transplante do ovário ou fragmentos fora da pélvis, abdômen, braço, etc.

- Experimental

\section{Ética e garantias de sucesso}

As associações médicas internacionais de medicina reprodutiva muitas vezes questionam o valor destas técnicas de preservação da fertilidade, devido a garantias restritas de sucesso de gravidez após o término do tratamento oncológico. Entretanto, só após o paciente ou responsáveis por ele conhecerem as reais vantagens deste procedimento é que deverá ser tomada a decisão definitiva. As taxas de sucesso são variáveis e podem chegar até $40 \%$, cabendo ao congelamento de sêmen e embriões a maior possibilidade de resultados positivos, pois estas técnicas, ao contrário das outras, já são utilizadas há muitos anos. As mais recentes técnicas, como o congelamento de óvulos, têm demonstrado taxas de gravidez ao redor de $30 \%$, e o congelamento de ovário e fragmentos de testículo com resultados ainda discretos. É importante lembrarmos que o futuro promete novos 


\section{ALGORITMO PARA PRESERVAÇÃO DA FERTILIDADE EM MULHERES COM CÂNCER}

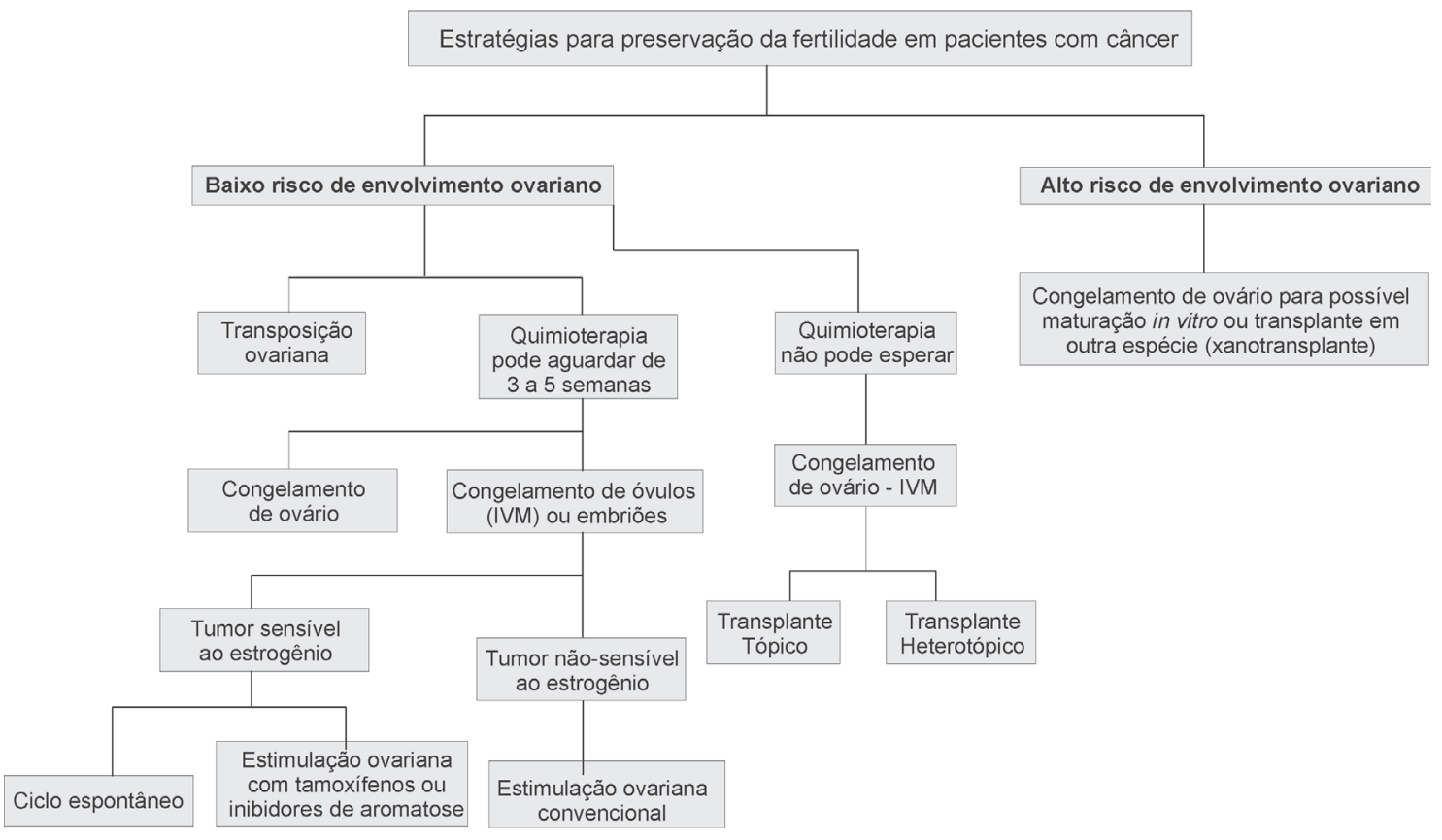

avanços que permitirão alcançar melhores resultados e talvez coincidam com a época que o paciente deseje ter seus filhos. Nós, particularmente concordamos com Revel e Shenker (2004) que, em um debate publicado pela revista Human Reproduction, defendeu a idéia de oferecer o congelamento de tecido ovariano a estas pacientes, antes do início da quimioterapia, quando a indução da ovulação não for possível. Lembra-se aqui que medicina é considerada uma "ciência de meios" e não uma "ciência de fins". Por isto devem ser citados os princípios do Código de Ética Médica.

\section{Capítulo I}

Artigo 5-O médico deve aprimorar continuamente seus conhecimentos e usar o melhor progresso em benefício do paciente.

\section{Capítulo $V$}

Artigo 57 - Não deixar de utilizar todos os meios disponíveis de diagnóstico e tratamento a seu alcance em favor do paciente.

A procriação deve ser um direito de todos e é reconhecido na Declaração Universal dos Direitos do Homem (Resolução da III Sessão Ordinária da Assembléia Geral das Nações Unidas aprovada em Paris em 10 de dezembro de 1978). Nesta declaração destaca-se que, além da igualdade e da dignidade, o ser humano tem direito de fundar uma família (Declaração Universal dos Direitos do Homem, artigos III, VII e XVI, 1) - Fonte: Reprodução Assistida: até onde podemos chegar? - Alvarez de Oliveira e Borges Jr.

Muitas vezes precisamos olhar o futuro e lembrar da rapidez do desenvolvimento da ciência e aí ponderar, refletir e imaginar a reação de um paciente, recuperado do seu câncer, e ao saber que antes do início do seu tratamento, não lhe foi oferecido qualquer chance de preservar a sua fertilidade. Uma oportunidade única que para alguns pode ser mínima, mas que pode garantir no futuro $100 \%$ de resultados positivos. Este conceito de proporções de sucesso é sobejamente conhecido pelos pacientes e familiares que já passaram pelas possíveis dúvidas sobre o diagnóstico e tratamento desta doença e, por isto, já estão acostumados com este tipo de dificuldade. A cada dia e a cada ano os resultados de recuperação da fertilidade pelas células e tecidos preservados estão maiores, cabendo ao profissional responsável ponderar com seu paciente ou familiares, caso seja ainda adolescente, como direcionar seu futuro reprodutivo.

\section{Abstract}

In recent years, technological advances in the area of oncology have offered treatment to patients increasing the expectation of a better life. Young cancer patients, who often had a grim future, can now look forward to being cured of their disease. However, this development has not always prevented the loss of reproductive health caused by the surgery, chemotherapy or radiotherapy, frequently used in the cure of these patients. Experts in Oncology and Hematology should be aware of the existence of techniques that can preserve fertility and be able to discuss this matter with their patients prior to treatment. New fertility preservation techniques such as oocyte freezing, ovarian and testicular fragment freezing, and other established techniques such as sperm and embryo cryopreservation when associated with assisted fertilization, open the prospect of pregnancy after treatment. Thus, no longer does the desire to have 
children need to be a frustration but can be a viable reality for patients undergoing oncological treatments. Rev. Bras. Hematol. Hemoter. 2008;30(5):406-410.

Key words: Fertility preservation; assisted reproduction; oocyte freezing; oncology.

\section{Referências Bibliográficas}

1. Blatt J. Pregnancy outcome in long-term survivors of childhood cancer. Med Pediatr Oncol. 1999;33(1):29-33

2. Jemal A, Murray T, Samuels A, et al. Cancer statistics, 2003. CA Cancer J Clin. 2003;53(1):5-26.

3. Oktay KH, Yih M. Preliminary experience with orthotopic and heterotopic transplantation of ovarian cortical strips. Semin Reprod Med. 2002;20(1):63-74.

4. Bleyer WA. Cancer in older adolescents and young adults: epidemiology, diagnosis, treatment, survival, and importance of clinical trials. Med Pediatr Oncol. 2002;38(1):1-10.

5. Larsen EC, Schmiegelow K, Rechnitzer C, et al. Radiotherapy at a young age reduces uterine volume of childhood cancer survivors. Acta Obstet Gynecol Scand. 2004;83(1):96-102.

6. Meirow D, Nugent D. The effects of radiotherapy and chemotherapy on female reproduction. Hum Reprod Update. 2001; 7(6):535-43.

7. Littley MD, Shalet SM, Morgenstern GR, Deakin DP. Endocrine and reproductive dysfunction following fractionated total body irradiation in adults. Q J Med. 1991;78(287):265-74.

8. Chapman RM, Sutcliffe SB, Malpas JS. Male gonadal dysfunction in Hodgkin's disease. A prospective study. J Am Med Assoc. 1981; 245(13):1323-8.

9. Vigersky RA, Chapman RM, Berenberg J, Glass AR. Testicular dysfunction in untreated Hodgkin's disease. Am J Med. 1982;73 (4):482-6.
10. Wood JW. Fecundity and natural fertility in humans. Oxford Rev Reprod Biol. 1989;11:61-109.

11. Menken J, Trussell J, Larsen U. Age and infertility. Science. 1986; 233(4771):1389-94.

12. Kuwayama M, Vajta G, Kato O, Leibo SP. Highly efficient vitrification method for cryopreservation of human oocytes. Reprod Biomed Online. 2005;11(3):300-8.

13. Oktay K, Economos K, Kan M, et al. Endocrine function and oocyte retrieval after autologous transplantation of ovarian cortical strips to the forearm. J Am Med Assoc. 2001;286(12): 1490-33.

14. Tan SL, Child TJ, Gulekli B. In vitro maturation and fertilization of oocytes from unstimulated ovaries: predicting the number of immature oocytes retrieved by early follicular phase ultrasonography. Am J Obstet Gynecol. 2002;186(4):684-9.

15. Toth TL, Baka SG, Veeck LL, et al. Fertilization and in vitro development of cryopreserved human prophase I oocytes. Fertil Steril. 1994;61(5):891-4.

16. Morice P, Juncker L, Rey A, et al. Ovarian transposition for patients with cervical carcinoma treated by radiosurgical combination. Fertil Steril. 2000;74(4):743-8.

17. Tulandi T, Al-Took S. Laparoscopic ovarian suspension before irradiation. Fertil Steril. 1998;70(2):381-3.

18. Oktay K. Ovarian tissue cryopreservation and transplantation: preliminary findings and implications for cancer patients. Hum Reprod Update. 2001;7(6):526-34

19. Waggoner SE. Cervical cancer. Lancet. 2003;361(9376):2217-25.

Avaliação: Editor e dois revisores externos

Conflito de interesse: não declarado

Recebido: 07/02/2008

Aceito: 26/02/2008 